\title{
Analysis of circulating nutritional antigen-specific T-cells in celiac disease and inflammatory bowel disease
}

\author{
Rainer Glauben ${ }^{1}$, Yasmina Rodriguez-Sillke ${ }^{1}$, Michael Schumann ${ }^{1}$, Donata Lissner ${ }^{1}$, \\ Federica Branchi ${ }^{1}$, Ulrich Steinhoff ${ }^{2}$, and Britta Siegmund ${ }^{1}$ \\ ${ }^{1}$ Charite Universitatsmedizin Berlin \\ ${ }^{2}$ Philipps University Marburg Faculty of Medicine
}

October 22, 2020

\begin{abstract}
Background: The present study aims to detect, quantify and analyze circulating nutritional antigen-specific T-cells in patients with celiac disease (CeD) as well as inflammatory bowel disease (IBD), thus comparing the specific T-cell response following barrier disruption and antigen translocation. Methods: The antigen-reactive T-cell enrichment (ARTE) technique was applied allowing for a phenotypical and functional flow cytometric analysis of rare nutritional antigen-specific T-cells, including the CeD-causing gliadin (gluten), in the peripheral blood. Results: Our study indicates that by applying the ARTE technique, differences of gluten-specific T-cells as well as the differential cytokine expression between the patient groups can be detected, even without the burdening gluten re-exposure of the patients. CeD patients, independent from the presence or absence of gluten exposure in their current diet, featured an increase of the frequency of gliadin-specific T-cells, which were characterized by a pro-inflammatory phenotype. However, only for active CeD and a consecutive small intestinal barrier breach, an increase of distinct nutritional T-cells could be detected. Accordingly, frequency as well as pro-inflammatory phenotype of nutritional antigen-specific $\mathrm{T}$ cells were highest in Crohn's disease patients with small intestinal inflammation whereas no significant increase was observed in ulcerative colitis. Conclusion: In summary, the ARTE method allows not only for detection but also for functional analysis of these rare cells even in healthy subjects. Applying this method, we were able to demonstrate that for non-CeD-related nutritional antigens, small intestinal barrier breach is mandatory for a peripheral antigen-specific T-cell.
\end{abstract}

\section{Analysis of circulating nutritional antigen-specific T-cells in celiac disease and inflammatory bowel disease}

Running title: Antigen-specific T-cells in IBD

Yasmina Rodríguez-Sillke ${ }^{1,2}$, Michael Schumann ${ }^{1}$, Donata Lissner ${ }^{1}$, Federica Branchi ${ }^{1}$, Ulrich Steinhoff ${ }^{3}$, Britta Siegmund $^{1 *}$, Rainer Glauben ${ }^{1 *}$

${ }^{1}$ Charité - Universitätsmedizin Berlin, corporate member of Freie Universität Berlin, Humboldt-Universität zu Berlin and Berlin Institute of Health, Medizinische Klinik für Gastroenterologie, Infektiologie und Rheumatologie, Berlin, Germany

${ }^{2}$ University of Potsdam, Institute of Nutrition, Nuthetal, Germany

${ }^{3}$ Institute for Medical Microbiology and Hospital Hygiene, Philipps University of Marburg, Germany.

* These authors contributed equally to this work.

\section{Corresponding author:}

Rainer Glauben, $\mathrm{PhD}$ 
Charité - Universitätsmedizin Berlin, Campus Benjamin Franklin

Medizinische Klinik für Gastroenterologie, Infektiologie, Rheumatologie

Hindenburgdamm 30, 12200 Berlin, Germany

E-mail: rainer.glauben@charite.de

Contributions : YRS performed and analyzed the experiments, DL and FB provided samples and patient information, YRS, MS, US, BS and RG designed the experiments, interpreted the data and authored the manuscript.

Acknowledgment: We thank Inka Freise for her technical assistance. This work was funded by the Deutsche Forschungsgemeinschaft SPP1656 (SPP1656); TRR241 (BS), DFG-SI749/10-1, and the Deutsche ZöliakieGesellschaft e.V. (BS, RG and YRS).

Conflict of Interests : No conflicts of interest exist for any of the coauthors.

\section{Abstract:}

Background: The present study aims to detect, quantify and analyze circulating nutritional antigen-specific T-cells in patients with celiac disease (CeD) as well as inflammatory bowel disease (IBD), thus comparing the specific T-cell response following barrier disruption and antigen translocation.

Methods: The antigen-reactive T-cell enrichment (ARTE) technique was applied allowing for a phenotypical and functional flow cytometric analysis of rare nutritional antigen-specific T-cells, including the CeD-causing gliadin (gluten), in the peripheral blood.

Results: Our study indicates that by applying the ARTE technique, differences of gluten-specific T-cells as well as the differential cytokine expression between the patient groups can be detected, even without the burdening gluten re-exposure of the patients. $\mathrm{CeD}$ patients, independent from the presence or absence of gluten exposure in their current diet, featured an increase of the frequency of gliadin-specific T-cells, which were characterized by a pro-inflammatory phenotype. However, only for active $\mathrm{CeD}$ and a consecutive small intestinal barrier breach, an increase of distinct nutritional T-cells could be detected. Accordingly, frequency as well as pro-inflammatory phenotype of nutritional antigen-specific T cells were highest in Crohn's disease patients with small intestinal inflammation whereas no significant increase was observed in ulcerative colitis.

Conclusion: In summary, the ARTE method allows not only for detection but also for functional analysis of these rare cells even in healthy subjects. Applying this method, we were able to demonstrate that for nonCeD-related nutritional antigens, small intestinal barrier breach is mandatory for a peripheral antigen-specific T-cell.

\section{Keywords:}

antigen-specific T-cells / celiac disease / gluten / IBD / nutritional antigens

word count: 2648

\section{Introduction}

Antigen-specific T-cells play a central role in the adaptive immune system, promoting specific acute immune responses and the formation of immunological memory. Analyzing not only frequency, but also phenotype and function of these rare cells, represents a critical step to understand the mechanisms of adaptive immunity in general, but also to determine the specific immune status of the individual patient or to diagnose infectious or auto-immune diseases. The high diversity of the T-cell receptor, which allows for recognition of billions of different antigens, leads to an extremely low frequency of T-cells, specific for a single peptide-MHC ligand. This holds true even for pathogen-specific memory compartments in the absence of acute infections, for which specific T-cell frequencies in peripheral blood are typically far below 1\%, but all the more for the naive repertoire $(<0.0005 \%)^{1,2}$. Hence, the analysis of antigen-specific T-cells demands methods which are 
highly specific, but in parallel allow processing of large cell numbers to enable quantification of these rare events. For the last decade, techniques such as antigen-specific proliferation via ${ }^{3} \mathrm{H}$-thymidine incorporation ${ }^{3}$ were applied, where antigen-specific T-cells have been analyzed via bulk stimulation of peripheral blood mononuclear cells (PBMC) with the respective antigen. However, in such assays, it is not feasible to detect the phenotypic and functional properties of the original reactive cells and therefore it is almost impossible to determine the actual frequency and phenotype of these cells in the starting cell sample or to exclude bystander effects. ELISPOT ${ }^{4}$, as an additional technical approach, reveals information on a single cell level, but lacks the high throughput capabilities to detect and quantify rare cells to an extent, where for example food antigen-specific T-cells in healthy individuals can be studied. Several studies aimed to characterize the systemic T-cell response to decipher the role of circulating T-cells and their functional impact ${ }^{5,6}$. Until now, the specific analysis of gluten-specific T-cells via flow cytometry depended on tetramer analysis ${ }^{5}$, which is limited to the knowledge and availability of the antigen epitope ${ }^{7}$, but also the frequency of target cells, therefore suffering from a high variability in cytokine analysis ${ }^{8}$.

Among autoimmune diseases, celiac disease $(\mathrm{CeD})$ represents a model disease, since gluten has been identified as the disease-causing antigen and elimination of gluten results in regeneration of the duodenal mucosa and consecutive wellbeing of the patient ${ }^{9}$. However, the diagnosis in $\mathrm{CeD}$ patients who are already on a gluten-free diet (GFD) remains challenging. Under a gluten-free diet (GFD), tissue-transglutaminase (tTG) antibodies normalize and the small intestinal villus atrophy regenerates. Until now, a burdening re-challenge of the patients to gluten is mandatory for a valid diagnosis. Yet, translocation of nutritional (and pathogenic) antigens due to intestinal barrier breaches is described not exclusively for $\mathrm{CeD}$, but also for inflammatory bowel diseases (IBD). Although there are first studies connecting GFD to improvement of patient wellbeing ${ }^{10}$ and even microbiota composition ${ }^{11}$ in IBD, until now, circulating nutritional antigen-specific T-cells have not been analyzed in these patients. Since the small intestine is the primary contact surface for food antigens and hence for the immunological response, we analyzed the specific nutritional T-cell response in the peripheral blood of patients with small intestinal Crohn's disease (CD), celiac disease as well as ulcerative colitis (UC), respectively. To exclude the influence of a non-intestinal inflammation, rheumatoid arthritis patients (RA) were included as control.

We here applied the novel antigen-reactive T-cell enrichment (ARTE) ${ }^{7}$ technology to determine the specific nutritional effector T-cell response in the peripheral blood. The ARTE technique is based on the stimulation of PBMC with a defined antigen and the subsequent up-regulation of the activation marker CD154 ${ }^{+}$, which is exclusively expressed on antigen-specific CD4 ${ }^{+} \mathrm{T}$-cells ${ }^{12}$. This method permits the detection of the entire antigen-specific CD4 ${ }^{+}$T-cell response just by adding the antigen of choice directly to PBMC without the need of in-vitro expanding the reacting cells. The subsequent enrichment of CD154 ${ }^{+}$cells enables further indepth phenotyping of this rare cell population. Thus, the ARTE technique allows a direct ex vivo cytometric - and hence functional - analyses of gluten-specific, but also even rarer nutritional antigen-specific T-cells.

\section{Methods}

PBMC from CeD, CD, UC and rheumatoid arthritis patients as well as healthy controls (Table 1 ) were cultured for $6 \mathrm{~h}$ in the presence of defined antigens followed by magnetic enrichment of activated CD154 T-cells (as marker for antigen-specific T-cells) ${ }^{7}$ (Figure 1A, B ).

\section{Blood donors and PBMC isolation}

Peripheral blood samples were obtained from healthy donors and patients of the Charité - Universitätsmedizin Berlin, Medical Department, Division of Gastroenterology, Infectiology and Rheumatology. All blood donors gave informed consent and the study was approved by the ethical committee of the Charité - Universitätsmedizin Berlin. PBMC were freshly isolated from $20 \mathrm{~mL}$ blood by density gradient centrifugation (Biocoll; Biochrom, Berlin, Germany). Heparinized whole blood was layered on the Biocoll Separation Solution and centrifuged at $1200 \mathrm{~g}$ for $25 \mathrm{~min}$ at $21{ }^{\circ} \mathrm{C}$. PBMC were collected from the interphase, washed and resuspended in RPMI1640 (Gibco, Life Technologies, Darmstadt, Germany) supplemented with 5\% human AB-serum (Sigma-Aldrich, St. Louis, MO, USA). 


\section{Patients}

PBMC of CD patients with small intestinal manifestation and UC patients with either active disease (defined by Harvey Bradshaw Index (HBI)) or remission (defined by HBI; pMayo) ${ }^{13,14}$ were analyzed. Additionally, $\mathrm{CeD}$ patients on a GFD for at least one year, newly diagnosed active $\mathrm{CeD}$ patients still exposed to gluten (aCeD), or GFD-refractory CeD patients (Refr) were included. Moreover, healthy first-degree relatives (FDR) on a regular diet without symptoms were included. The diagnosis of $\mathrm{CeD}$ was based on the presence of tTG antibodies in the serum and characteristic histopathological features in duodenal biopsies (Marsh score $>1$ ). Refractory celiac disease diagnosis was based on the presence of a Marsh III enteropathy and clinical malabsorption in spite of consumption of a gluten-free diet for at least one year. Clonality analysis as done by PCR of the CDR3 region of the TCR was performed. Detection of a clonal T-cell population and aberrant lymphocytes by immune phenotyping of duodenal tissue allowed for diagnosis of Refr. type II. All other Refr. cases were diagnosed as RCD type $\mathrm{I}^{15}$. HLA-DQ status could not be determined for IBD patients and controls. As additional non-intestinal inflammatory control group PBMC from rheumatoid arthritis (RA) patients were analyzed.

\section{Antigen-reactive T-cell enrichment}

Identification and enrichment of antigen-reactive T-cells was performed by applying the recently described ARTE technique ${ }^{7}$. Briefly, $0.5-1 \times 10^{7}$ PBMC were cultured in RPMI1640 supplemented with $5 \%$ human AB-serum and stimulated for $6 \mathrm{~h}$ with $1 \mu \mathrm{g} / \mathrm{mL}$ CD40 (Miltenyi Biotec, Bergisch Gladbach, Germany) in the presence or absence of the pepsin-trypsin digested 33-mer gliadin peptide (200 $\mu \mathrm{g} / \mathrm{mL}$ ) (Sigma- Aldrich), OVA-peptide (Invitrogen) or soybean or peanut extract $(200 \mu \mathrm{g} / \mathrm{mL})$ (Greer Laboratories). For the last 2 $\mathrm{h}, 1 \mu \mathrm{g} / \mathrm{mL}$ brefeldin A (Sigma- Aldrich) was added. Cells were indirectly labeled with anti-CD154-biotin antibody, followed by anti-biotin MicroBeads (CD154 MicroBead-Kit, Miltenyi Biotec) and magnetically enriched using MS columns (Miltenyi Biotec).

\section{Flow cytometric cell analysis}

Surface staining was performed on the MS column (first panel: Brilliant violet 510 anti-human CD4; RPAT4, Brilliant Violet 421 anti-human CD197 (CCR7); G043H7, PE/Cy7 anti-human CD45RA; HI100, second panel: Brilliant violet 510 anti-human CD4; PE/Cy7 anti-human CD29/B1; TS2/16, PE anti-human B7; FIB504, all from BioLegend (Koblenz, Germany), VioBlue anti-human CD49d/a4; MZ18-24A9 from Miltenyi Biotec. (Bergisch Gladbach, Germany) and human FC block (CSL Behring, Marburg, Germany)). The enriched cell fraction was fixed using eBioscience, FoxP3 staining buffer, Thermo Fisher Scientific, Waltham, MA USA) and intracellular staining was performed (APC anti-human IFN $\gamma \cdot 4 \mathrm{~S}$.B3, APC/Cy7 anti-human IL-17A; BL168, PerCP/Cy5.5 anti-human TNF $\alpha$ MAb11, all from BioLegend and FITC anti-human CD154 (5C8) from Miltenyi Biotec. Flow cytometry analysis was performed on the FACS Canto II device (BD Bioscience, Heidelberg, Germany). Data were analyzed with FlowJo analysis software (Ashland, OR, USA).

\section{Statistics}

Statistical analysis was performed using Prism software (GraphPad Software). Significance was determined using Mann-Whitney-U-Test as indicated. ${ }^{*} \mathrm{P}>0.05,{ }^{* *} \mathrm{P}>0.01,{ }^{* * *} \mathrm{P}>0.001$.

\section{Results}

\section{Circulating gliadin-specific T-cells are increased in active disease with ileal inflammation}

ARTE technology was applied to all blood samples (Figure 1A ) for various nutritional antigens, including controls for antigen-specific T-cell enrichment and T-cell activation. Moreover, we could clearly demonstrate the necessity for T-cell enrichment to allow for deeper cell analysis and therefore the advantage of this method over direct staining protocols for rare antigen-specific cell populations (Figure 1B ). With the overall frequency of $\mathrm{CD} 4^{+}$T-cells remaining stable in the various disease conditions (Figure 1C ), the frequency of gliadin-specific CD154 ${ }^{+}$T-cells among CD4 ${ }^{+}$T-cells in PBMC was expectedly highest in active $\mathrm{CeD}(\mathrm{aCeD})$, i.e. without GFD, as well as in refractory CeD patients (Refr). aCeD are rare patients as we did 
not actively initiate a gluten-re-challenge. Moreover the frequencies were also significantly increased in $\mathrm{CeD}$ patients on a GFD without clinical symptoms, when compared to healthy controls. Remarkably, a similar frequency to active $\mathrm{CeD}$ patients was observed in active $\mathrm{CD}$ patients with ileal inflammation (Figure 2A ).

The frequency was significantly lower in CD patients in remission, in UC patients, independent of their inflammatory state and in healthy controls. Interestingly, first-degree relatives (FDR) of CeD patients, considered healthy by standard diagnostics, revealed a significant increase in the frequency of gliadin-specific T-cells compared to controls without familiar predisposition of CeD. Of notice, RA as auto-inflammatory control without intestinal inflammation, did not differ from healthy controls.

\section{Phenotyping circulating gliadin-specific T-cells}

Further phenotyping of gliadin-specific CD4 ${ }^{+} \mathrm{CD} 154^{+}$T-cells in effector memory (CD45RA- $\mathrm{CCR}^{-}$), central memory $\left(\mathrm{CD} 45 \mathrm{RA}^{-} \mathrm{CCR} 7^{+}\right)$or naïve $\left(\mathrm{CD} 45 \mathrm{RA}^{+} \mathrm{CCR} 7^{+}\right)$cells revealed a shift towards the memory types in the groups with active small intestinal inflammation. Remarkably, effector memory T-cells outnumbered naïve T-cells (ratio $>1$, Figure 2B ) only in aCeD, Refr and CD patients. Moreover, gliadin-specific CD4 ${ }^{+} \mathrm{CD} 154^{+}$ T-cells, positive for the small intestinal homing marker $\alpha 4 \beta 1$, but not for $\alpha 4 \beta 7$, a general gut homing marker, were increased in aCeD patients (Figure 2C, D ), further strengthening the connection of peripheral nutritional antigen-specific T-cells to small intestinal inflammation.

\section{Pro-inflammatory cytokines of circulating gliadin-specific T-cells}

The subsequent functional analysis of the antigen-specific T-cells after gliadin stimulation (Figure 2E, F; S. Figure 1 ) revealed highest production of the pro-inflammatory cytokines IFN $\gamma$, IL-17A and TNF $\alpha$ in cells from aCeD, Refr, but also from CD patients with small intestinal involvement. Remarkably, antigen-specific T-cells of first-degree relatives of CeD patients (FDR) presented with higher frequencies of gliadin-specific T-cells and an increased TNF $\alpha$ expression, and were thus comparable to aCeD patients. TNF $\alpha$-positive $\mathrm{CD} 154^{+}$cells were most discriminative when comparing active and inactive $\mathrm{CeD}$ to healthy controls.

\section{Further food antigen-specific T-cells are increased in active disease with ileal inflammation}

To dissect a sole barrier defect, as it is present in small intestinal CD, from the disease-driving gliadinreactivity in $\mathrm{CeD}$, we included soybean protein, peanut protein and OVA-peptide in our analysis. In fact, an increased frequency of antigen-specific $\mathrm{CD} 4^{+} \mathrm{T}$-cells was exclusively observed in the presence of small intestinal inflammation, namely $\mathrm{CD}$ and $\mathrm{aCeD}$. CD patients in remission as well as UC patients independent of their inflammatory state did not differ from healthy controls. Furthermore, CeD patients on GFD, although being highly reactive to gliadin, showed no reaction to other nutritional antigens (Figure 3A, B ) .

\section{Discussion}

So far, the published data for ARTE have been focusing on bacteria- or fungi-specific antigens as well as house dust mites ${ }^{16}$. However, this methods allows also to study even rarer nutritional antigen-specific T-cells without in-vitro expanding of the reacting cells and without re-challenging the patients. Therefore, we apply this method for detection of the rare nutritional antigen-specific T-cells in peripheral blood, in order to analyze antigen reactivity for different clinical subgroups of CeD and IBD patients.

Recently, peripheral gluten-specific CD4 ${ }^{+}$T-cells were analyzed applying HLA-DQ2:gluten tetramers, thus identifying an increase of gluten-specific $\mathrm{CD} 4^{+} \mathrm{T}$-cells in $\mathrm{aCeD}^{5,6}$. However, the here applied ARTE allows for a deeper analysis of the respective specific $\mathrm{CD} 4^{+} \mathrm{T}$-cells to distinguish different disease states of CeD. The diagnosis of $\mathrm{CeD}$ in patients who already follow a GFD is challenging, since tTG antibodies under GFD normalize and histological markers, as the small intestinal villous atrophy, regenerates. This clinical need is even growing, giving the increasingly popular gluten-free life style in the western world ${ }^{17,18}$, or for first degree relatives, who frequently initiate a GFD when a household member is diagnosed with CeD. For the latter, the high risk of developing $\mathrm{CeD}$ has been proven in many studies ${ }^{19,20}$ and surveillance for $\mathrm{CeD}$ is even recommended for first-degree relatives of a diagnosed patient where carriage of a risk gene has not been 
excluded ${ }^{21,22}$. Work herein might be the first step towards identifying such cases, without a conventional burdening gluten re-challenge, since characteristic changes in cytokine expression in gliadin-specific CD4 ${ }^{+}$ T-cells in the peripheral blood are present. For the rare subgroup of Refr patients, especially for type I, the specific immunological nature remains unclear. Diagnosis is still based on histopathology alone, while recent studies suggest a heterogeneous composition of different pathologies to be merged under this term. In this respect, the ARTE technique for gliadin-specific T-cells represents a unique research tool for future studies that has the potential to contribute to a subclassification of this disease group. Furthermore, our data reveal a specific immunological phenotype of gliadin-specific $\mathrm{CD} 4^{+} \mathrm{T}$-cells from FDR regarding the hypersensitivity towards gluten, even if diagnosed as healthy due to their tTG status (without GFD). By demonstrating an active immune response against the pathogenic antigen, identification and even phenotyping of glutenreactive T-cells from peripheral blood might represent an alternative, all the more in pediatric cases, where first time diagnosis is common, while invasive endoscopy is meant to be avoided. Thus, this novel approach could fulfill the clinical need for a noninvasive marker of $\mathrm{CeD}$ activity as clinical and research tool ${ }^{23}$.

With regard to IBD, which shares the characteristics of barrier disruption ${ }^{24}$ and subsequent intestinal inflammation in the lamina propria, we detected increased levels of gliadin-specific T-cells in the peripheral blood of active CD patients with concurrent small intestinal inflammation, paralleled by the highest frequency of antigen-specific T-cells expressing pro-inflammatory cytokines. This distinct occurrence suggests the small intestinal barrier disruption as major cause for the observed T-cell activation, since these cells express small intestinal homing markers. The homing to the ileum ( $\alpha 4 \beta 1)$ was described as essential pathway in $\mathrm{CD}{ }^{25}$. In line, only in these three patient groups of active small intestinal inflammation, the effectormemory T-cells outnumbered the naïve phenotype among gliadin-specific T-cells in the peripheral blood. Furthermore, peripheral T-cells from CD patients with small intestinal inflammation proved to be responsive to other major nutritional antigens ${ }^{26}$, while neither active UC nor CD or UC in remission showed any reaction. Again, only antigen-specific T-cells from active CeD, but not GFD patients demonstrated similar properties, corroborating on the one hand the leaky barrier of the affected small intestine as the site of food antigen translocation and subsequent T-cell activation. On the other hand, the significant effect of gliadin, but no other food antigen, in the GFD group further confirmed the singular antigen-driven nature of $\mathrm{CeD}$. Nevertheless, based on surveys, it has been suggested, that long-term GFD improves gastrointestinal symptoms in active IBD patients ${ }^{10}$. With the present study, we are able to convey cellular and functional data by demonstrating an enhanced gliadin-specific response of pro-inflammatory cytokines towards gliadin for CD patients

Our data strongly suggest, that small intestinal inflammation is key for the development of a nutritional antigen-specific T-cell response. Therefore, ARTE allows to distinguish CD with small intestinal inflammation from $\mathrm{UC}$ and $\mathrm{CD}$ in remission by a unique profile of circulating antigen-specific T-cells, raising the question, if a well-defined nutritional regimen (e.g. GFD) might have therapeutic potential in the setting of IBD. Hence, based on the analysis of the systemic immune response, an "anti-inflammatory" diet could be developed and monitored. In addition, this technique allows detailed analyses of gliadin-specific T-cells in such a high resolution that even healthy first-degree relatives can be discriminated and might thus provide a novel noninvasive diagnostic tool to identify symptom-free $\mathrm{CeD}$ patients on a gluten-free diet.

\section{References}

1. Kwok WW, Tan V, Gillette L, et al. Frequency of epitope-specific naive CD4(+) T cells correlates with immunodominance in the human memory repertoire. J Immunol. 2012;188(6):2537-2544.

2. Chu HH, Moon JJ, Takada K, et al. Positive selection optimizes the number and function of MHCII-restricted CD4+ T cell clones in the naive polyclonal repertoire. Proc Natl Acad Sci $U S$ A.2009;106(27):11241-11245.

3. Gjertsen HA, Sollid LM, Ek J, Thorsby E, Lundin KE. T cells from the peripheral blood of coeliac disease patients recognize gluten antigens when presented by HLA-DR, -DQ, or -DP molecules. Scand $J$ Immunol.1994;39(6):567-574. 
4. Anderson RP, van Heel DA, Tye-Din JA, et al. T cells in peripheral blood after gluten challenge in coeliac disease. Gut.2005;54(9):1217-1223.

5. Sarna VK, Lundin KEA, Morkrid L, Qiao SW, Sollid LM, Christophersen A. HLA-DQ-Gluten Tetramer Blood Test Accurately Identifies Patients With and Without Celiac Disease in Absence of Gluten Consumption. Gastroenterology. 2018;154(4):886-896 e886.

6. Ben-Horin S, Green PH, Bank I, Chess L, Goldstein I. Characterizing the circulating, gliadin-specific CD4+ memory $\mathrm{T}$ cells in patients with celiac disease: linkage between memory function, gut homing and Th1 polarization. J Leukoc Biol. 2006;79(4):676-685.

7. Bacher P, Scheffold A. Flow-cytometric analysis of rare antigen-specific T cells. Cytometry A. 2013;83(8):692-701.

8. Pastore G, Carraro M, Pettini E, Nolfi E, Medaglini D, Ciabattini A. Optimized Protocol for the Detection of Multifunctional Epitope-Specific CD4(+) T Cells Combining MHC-II Tetramer and Intracellular Cytokine Staining Technologies. Front Immunol. 2019;10:2304.

9. Schuppan D, Junker Y, Barisani D. Celiac disease: from pathogenesis to novel therapies. Gastroenterology. 2009;137(6):1912-1933.

10. Herfarth HH, Martin CF, Sandler RS, Kappelman MD, Long MD. Prevalence of a gluten-free diet and improvement of clinical symptoms in patients with inflammatory bowel diseases. Inflamm Bowel Dis.2014;20(7):1194-1197.

11. Bonder MJ, Tigchelaar EF, Cai X, et al. The influence of a short-term gluten-free diet on the human gut microbiome. Genome Med. 2016;8(1):45.

12. Frentsch M, Arbach O, Kirchhoff D, et al. Direct access to CD4+ T cells specific for defined antigens according to CD154 expression. Nat Med. 2005;11(10):1118-1124.

13. Harvey RF, Bradshaw JM. A simple index of Crohn's-disease activity.Lancet. 1980;1(8167):514.

14. Schroeder KW, Tremaine WJ, Ilstrup DM. Coated oral 5-aminosalicylic acid therapy for mildly to moderately active ulcerative colitis. A randomized study. $N$ Engl J Med. 1987;317(26):1625-1629.

15. Felber J, Aust D, Baas S, et al. [Results of a S2k-Consensus Conference of the German Society of Gastroenterolgy, Digestive- and Metabolic Diseases (DGVS) in conjunction with the German Coeliac Society (DZG) regarding coeliac disease, wheat allergy and wheat sensitivity].Z Gastroenterol. 2014;52(7):711-743.

16. Bacher P, Kniemeyer O, Schonbrunn A, et al. Antigen-specific expansion of human regulatory T cells as a major tolerance mechanism against mucosal fungi. Mucosal Immunol. 2014;7(4):916-928.

17. Kim HS, Patel KG, Orosz E, et al. Time Trends in the Prevalence of Celiac Disease and Gluten-Free Diet in the US Population: Results From the National Health and Nutrition Examination Surveys 20092014.JAMA Intern Med. 2016;176(11):1716-1717.

18. Hager AS, Taylor JP, Waters DM, Arendt EK. Gluten free beer - A review. Trends Food Sci Tech. 2014;36(1):44-54.

19. Acharya P, Kutum R, Pandey R, et al. First Degree Relatives of Patients with Celiac Disease Harbour an Intestinal Transcriptomic Signature that Might Protect them from Enterocyte Damage. Clin Transl Gastroenterol. 2018;9(10):195.

20. Singh P, Arora S, Lal S, Strand TA, Makharia GK. Risk of Celiac Disease in the First- and Second-Degree Relatives of Patients With Celiac Disease: A Systematic Review and Meta-Analysis. Am J Gastroenterol. 2015;110(11):1539-1548. 
21. Hill ID, Dirks MH, Liptak GS, et al. Guideline for the diagnosis and treatment of celiac disease in children: recommendations of the North American Society for Pediatric Gastroenterology, Hepatology and Nutrition. J Pediatr Gastroenterol Nutr. 2005;40(1):1-19.

22. Husby S, Koletzko S, Korponay-Szabo IR, et al. European Society for Pediatric Gastroenterology, Hepatology, and Nutrition guidelines for the diagnosis of coeliac disease. J Pediatr Gastroenterol Nutr.2012;54(1):136-160.

23. Leffler DA, Schuppan D. Update on serologic testing in celiac disease. Am J Gastroenterol. 2010;105(12):2520-2524.

24. Martini E, Krug SM, Siegmund B, Neurath MF, Becker C. Mend Your Fences: The Epithelial Barrier and its Relationship With Mucosal Immunity in Inflammatory Bowel Disease. Cell Mol Gastroenterol Hepatol. $2017 ; 4(1): 33-46$.

25. Zundler S, Fischer A, Schillinger D, et al. The alpha4beta1 Homing Pathway Is Essential for Ileal Homing of Crohn's Disease Effector T Cells In Vivo. Inflamm Bowel Dis. 2017;23(3):379-391.

26. Tordesillas L, Berin MC, Sampson HA. Immunology of Food Allergy.Immunity. 2017;47(1):32-50.

Table 1

\begin{tabular}{|c|c|c|c|c|c|}
\hline & $\begin{array}{l}\text { Non-relative } \\
\text { controls } \\
(\mathrm{n}=24)\end{array}$ & $\begin{array}{l}\text { First-degree } \\
\text { relatives } \\
(n=6)\end{array}$ & $\begin{array}{l}\text { Celiac } \\
\text { disease on } \\
\text { GFD }(n=24)\end{array}$ & $\begin{array}{l}\text { Active celiac } \\
\text { disease }(n=9)\end{array}$ & $\begin{array}{l}\text { Refractory } \\
\text { celiac } \\
\text { disease* } \\
(\mathrm{n}=11)\end{array}$ \\
\hline $\begin{array}{l}\text { Age (mean } \pm \\
\text { SD) }\end{array}$ & $33.3 \pm 9.4$ & $34.5 \pm 9.5$ & $43.9 \pm 16.8$ & $47.3 \pm 12.4$ & $61.1 \pm 11.6$ \\
\hline Female $[\%]$ & 55 & 83 & 80 & 66 & 82 \\
\hline $\begin{array}{l}\text { tTG }(\text { mean } \pm \\
\text { SD) }[\mathrm{U} / \mathrm{mL}] \#\end{array}$ & $1.6 \pm 0.7$ & $1.4 \pm 0.4$ & $6.3 \pm 4.8$ & $114.2 \pm 70.9$ & $21.0 \pm 27.2$ \\
\hline$[\mathrm{CE}]$ & - & - & - & $\begin{array}{l}3428.1 \pm \\
1313.7\end{array}$ & - \\
\hline $\begin{array}{l}\text { Marsh grade - } \\
\text { IIIa at first } \\
\text { diagnosis - IIIb } \\
\text { - IIIc }\end{array}$ & - & - & 1094 & 332 & 622 \\
\hline $\begin{array}{l}\text { RCD type I } \\
/ \text { II [\%] }\end{array}$ & - & - & - & - & $63.6 / 36.4$ \\
\hline
\end{tabular}

\begin{tabular}{|c|c|c|c|c|c|c|}
\hline & $\begin{array}{l}\text { Non-relative } \\
\text { controls } \\
(\mathrm{n}=24)\end{array}$ & $\begin{array}{l}\text { Rheumatoid } \\
\text { arthritis } \\
(\mathrm{n}=5)\end{array}$ & $\begin{array}{l}\text { Crohn's } \\
\text { disease } \\
(\mathrm{n}=19+13)\end{array}$ & $\begin{array}{l}\text { Crohn's } \\
\text { disease } \\
\text { (remission) } \\
(n=10+4)\end{array}$ & $\begin{array}{l}\text { Ulcerative } \\
\text { colitis } \\
(\mathrm{n}=12+7)\end{array}$ & $\begin{array}{l}\text { Ulcerative } \\
\text { colitis } \\
\text { (remission) } \\
(\mathrm{n}=9+2)\end{array}$ \\
\hline $\begin{array}{l}\text { Age (mean } \\
\pm \mathrm{SD})\end{array}$ & $33.3 \pm 9.4$ & $49.4 \pm 10.8$ & $36.2 \pm 9.3$ & $41.4 \pm 13.9$ & $41.0 \pm 14.9$ & $42.0 \pm 15.1$ \\
\hline Female [\%] & 55 & 67 & 50 & 57 & 37 & 64 \\
\hline $\begin{array}{l}\text { Clinical } \\
\text { score - HBI }\end{array}$ & - & - & $5.1 \pm 2.7$ & $0.5 \pm 1.2$ & - & - \\
\hline $\begin{array}{l}\text { - partial } \\
\text { Mayo }\end{array}$ & - & - & - & - & $3.9 \pm 1.9$ & $1.0 \pm 1.0$ \\
\hline
\end{tabular}




\begin{tabular}{|c|c|c|c|c|c|c|}
\hline & $\begin{array}{l}\text { Non-relative } \\
\text { controls } \\
(\mathrm{n}=24)\end{array}$ & $\begin{array}{l}\text { Rheumatoid } \\
\text { arthritis } \\
(\mathrm{n}=5)\end{array}$ & $\begin{array}{l}\text { Crohn's } \\
\text { disease } \\
(\mathrm{n}=19+13)\end{array}$ & $\begin{array}{l}\text { Crohn's } \\
\text { disease } \\
\text { (remission) } \\
(\mathrm{n}=10+4)\end{array}$ & $\begin{array}{l}\text { Ulcerative } \\
\text { colitis } \\
(\mathrm{n}=12+7)\end{array}$ & $\begin{array}{l}\text { Ulcerative } \\
\text { colitis } \\
\text { (remission) } \\
(\mathrm{n}=9+2)\end{array}$ \\
\hline 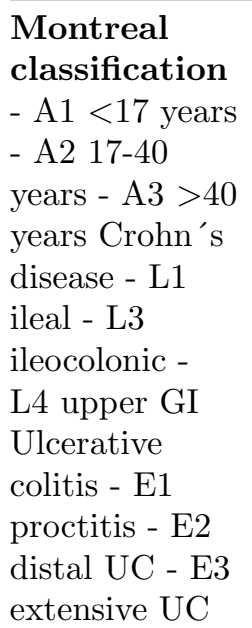 & - & - & 023911126 & 077482 & 07120810 & 065083 \\
\hline
\end{tabular}

HBI, Harvey-Bradshaw index

\section{Figure legends}

Figure 1. Enrichment of food antigen-specific T-cells.(A-C) Peripheral blood mononuclear cells (PBMC) were stimulated with various food antigens, magnetically enriched for CD154 and analyzed by flow cytometry. (A ) Methodology and (B ) exemplary density plots of CD154 ${ }^{+}$T-cell enrichment after stimulation with gliadin or control antigen are shown. (C ) Frequencies of CD4 ${ }^{+}$T-cells in PBMC were determined from healthy controls (non-relatives, NR), patients with active Crohn's disease (CD) or ulcerative colitis (UC), or each of these entities in remission (-R), celiac disease patients $(\mathrm{CeD}) \pm$ gluten-free diet $(\mathrm{GFD}$, aCeD) or refractory (Refr) patients, first degree relatives of $\mathrm{CeD}$ patients (FDR) and rheumatoid arthritis patients (RA). Data are shown as median with $95 \%$ CI.

Figure 2 . Phenotyping gliadin-specific T-cells.(A-F ) Peripheral blood mononuclear cells (PBMC) were stimulated with various food antigens, magnetically enriched for CD154 and analyzed by flow cytometry. (A ) Frequencies of CD154 $4^{+}$cells among CD4 ${ }^{+}$T-cells in PBMC from healthy controls (non-relatives, NR), patients with active Crohn's disease (CD) or ulcerative colitis (UC), or each of these entities in remission $(-\mathrm{R})$, celiac disease patients $(\mathrm{CeD}) \pm$ gluten-free diet $(\mathrm{GFD}, \mathrm{aCeD})$ or refractory (Refr) patients, first degree relatives of CeD patients (FDR) and rheumatoid arthritis patients (RA) are shown. (B ) Ratio of gliadinspecific effector memory CD4 ${ }^{+} \mathrm{CD} 154^{+}$T-cells $\left(\mathrm{CD} 45 \mathrm{RA}^{-} \mathrm{CCR} 7^{-}\right)$and naïve T-cells $\left(\mathrm{CD} 45 \mathrm{RA}{ }^{+} \mathrm{CCR} 7^{+}\right)$from controls and disease groups are shown. (C ) Exemplary dot plots of $\alpha 4 \beta 1$ within CD $4^{+}$and $\mathrm{CD} 4^{+} \mathrm{CD} 154^{+}$ T-cells are shown. (D ) Frequencies of CD $4^{+} \mathrm{T}$ cells and CD $4^{+} \mathrm{CD} 154^{+} \mathrm{T}$ cells, positive for integrins $\alpha 4 \beta 1$ and $\alpha 4 \beta 7$ are shown. (E ) Frequencies of gliadin-specific IFN $\gamma^{+}, \mathrm{IL}_{-17 \mathrm{~A}^{+}}$and $\mathrm{TNF} \alpha^{+}$cells within CD154 ${ }^{+}$ T-cells in between the patient groups are shown. (F ) Exemplary dot plots of IFN $\gamma^{+}, \mathrm{IL}_{-}-17 \mathrm{~A}^{+}$and $\mathrm{TNF} \alpha^{+}$ $\mathrm{CD} 4^{+} \mathrm{CD} 154^{+}$gliadin-specific T-cells are shown. Data are shown as median. Significance was determined using Mann-Whitney-U-Test. ${ }^{*} \mathrm{P}>0.05,{ }^{* *} \mathrm{P}>0.01,{ }^{* * *} \mathrm{P}>0.001$. Statistically significant differences were calculated in comparison to healthy non-relatives, if not indicated otherwise.

Figure 3 . Frequencies of food antigen-specific T-cells.(A-B ) Peripheral blood mononuclear cells (PBMC) were stimulated with various food antigens, magnetically enriched for CD154 and analyzed by flow cytometry. (A ) Exemplary dot plots of CD154 ${ }^{+}$T-cell enrichment of indicated nutritional antigens with absolute numbers and frequencies are shown. (B ) Frequencies of CD $154^{+}$cells among CD4 ${ }^{+}$T-cells after stimulation with 
soybean, peanut and OVA-peptide from healthy controls (non-relatives, NR), patients with active Crohn's disease (CD) or ulcerative colitis (UC), or each of these entities in remission (-R), celiac disease patients $(\mathrm{CeD}) \pm$ gluten-free diet (GFD, aCeD) or refractory (Refr) patients are shown. Data are shown as median. Significance was determined using Mann-Whitney-U-Test. ${ }^{*} \mathrm{P}>0.05,{ }^{*} \mathrm{P}>0.01$, ${ }^{* * *} \mathrm{P}>0.001$. Statistically significant differences were calculated in comparison to healthy non-relatives, if not indicated otherwise.

Figure 1

A

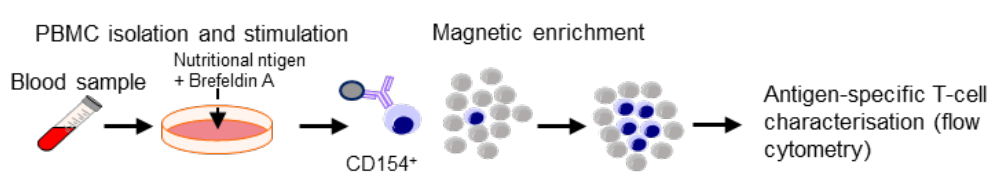

B

before enrichment after enrichment
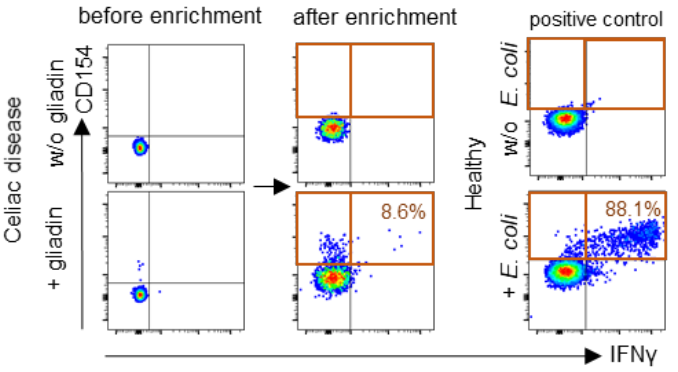

C

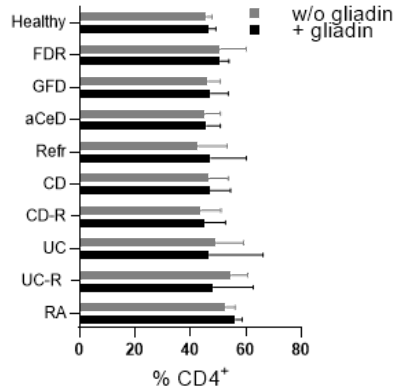

Figure 2: 
A Frequency of Gliadin-specific T-cells B

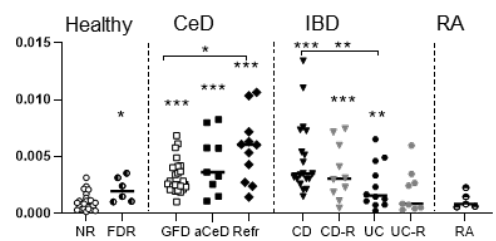

c

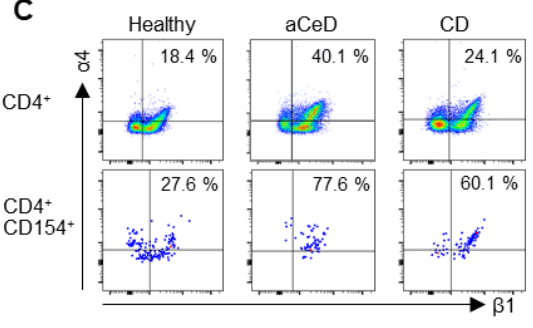

E cytokine expression of Gliadin-specific T-cells

F cytokine expression of Gliadin-specific T-cells
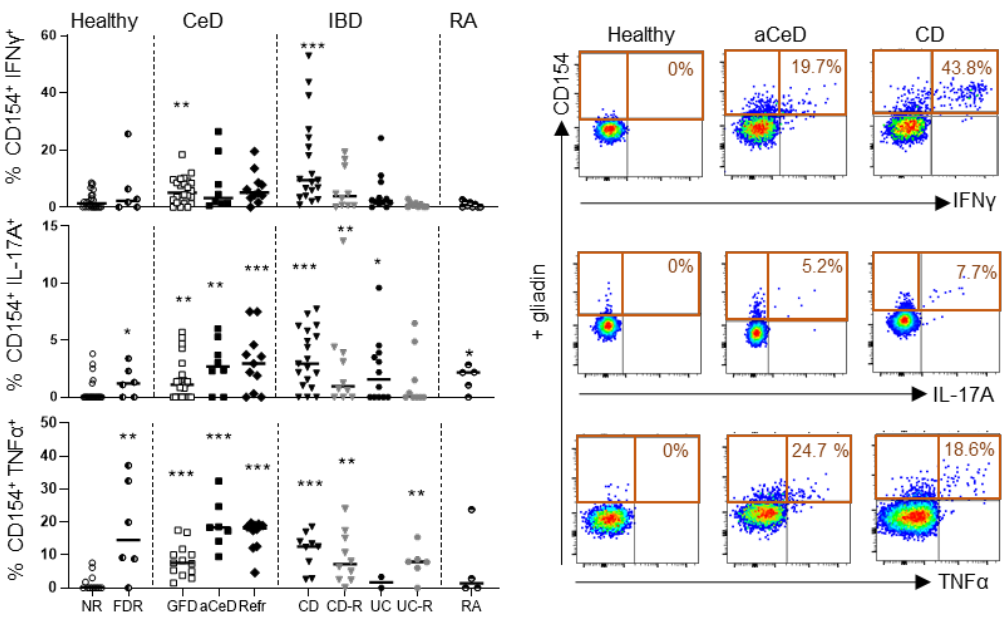

Figure 3: 
A

Active Crohn's disease

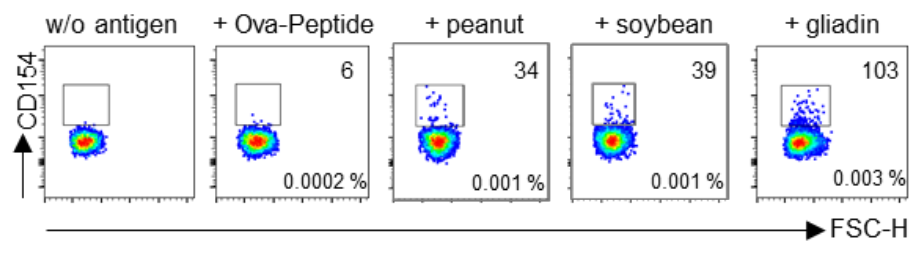

B

Soybeanspecific T-cells

Peanutspecific T-cells

Peanutspecific T-cells

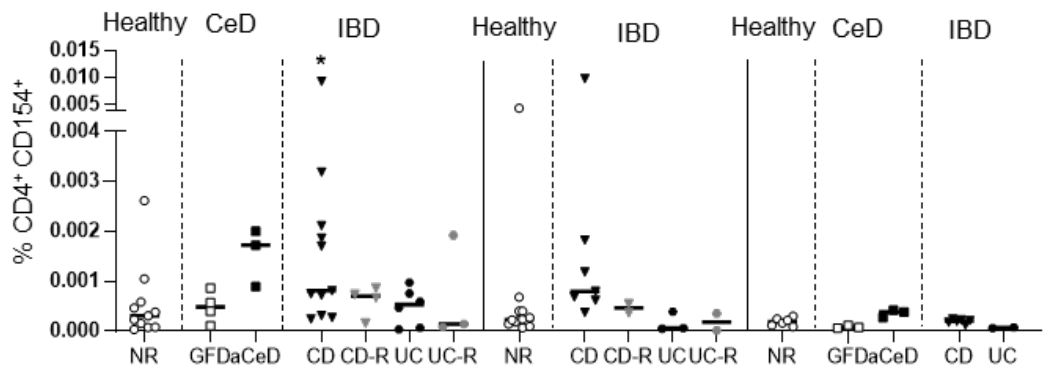

